High case volume and prior sigmoidoscopy experience are associated with a CIR $>90 \%$. The potential of both these factors to influence the attainment of competency should be exploited within endoscopy training programmes.

Disclosure of Interest None Declared.

\section{PTH-059 UPPER GASTROINTESTINAL MALIGNANCIES: 6976 ENDOSCOPY REVIEW IN A MULTINATIONAL STUDY}

doi:10.1136/gutjnl-2013-304907.546

${ }_{1,}^{1,}$ S Roy. 'Gastroenterology, Queen Mary's Hospital, Sidcup, UK

Introduction To compare the prevalence of upper gastrointestinal (UGI) malignant and premalignant conditions in three separate populations; British, Japanese and Arabian.

Methods 6976 Upper Gastrointestinal (UGI) endoscopies were retrospectively reviewed in a multinational comparative study. This involved three population groups: Group A- British \& others $(\mathrm{n}=2158)$; Group B- Japanese $(\mathrm{n}=2628)$; Group C- Arabians (Saudi Arabians \& others $(n=2190))$.

The majority of the patients fell in the above 16 age group.

The patients presented with UGI symptoms and were selected at random. The data was collected between 1986 and 2012

The study involved patients from Barnsley District General Hospital (Barnsley), Darent Valley Hospital (Dartford) \& Queen Mary's Hospital (Sidcup) in UK - Group A; Showa University Fujigaoka Hospital \& Niigata Cancer Centre Hospital (Japan)- Group B; Jubail Hospitals (Saudi Arabia) - Group C.

Results A comparison was made for the malignant and premalignant diseases. It revealed a very high incidence of UGI malignancies amongst Japanese (Group B). There is significant number of malignant diseases amongst non-Arabians, but such malignancies amongst Arabians are rare.

The incidence of gastric ulcers and gastric polyps are very high amongst Japanese compared to the other population groups in the study.

It is found that the prevalence of Barrett's Oesophagus has increased significantly in the last ten years.

The results are summarised as below:

UGI malignancies recorded:

Group A: British population: 37 (1.71\% with 95\% CI: 1.17 to 2.26) 6.51)

Group B: Japanese population: 148 (5.63\% with 95\% CI: 4.75 to

Group C: Arabian population: 16 (1.17\% with 95\% CI: 1.17 to 2.26)

Ulcers in the upper GI tract recorded:

Group A: British population: 366 (16.96\%, 95\% CI: 15.38 to 18.54)

Group B: Japanese population: 498 (18.95\% 95\% CI: 17.45 to 20.45)

Group C: Saudi population: $506(23.11 \%, 95 \%$ CI $=21.34$ to 24.87).

Conclusion It can be concluded that Arabians (Saudis) suffer rarely from UGI malignancies and it may be related to social, environmental, geographical, genetic and dietary habits. Dietary habits in Japan are very different from the British and Saudi population.

Alcohol consumption amongst Saudis is very little. In comparison, Japanese and UK populations drink alcohol significantly more.

Prevalence of Barrett's has increased significantly in the last 10 years. It is likely due to effective Helicobacter Pylori eradication and due to increasing awareness of Barrett's amongst endoscopists.

Disclosure of Interest None Declared.

\section{PTH-060 ACUTE UPPER GASTROINTESTINAL BLEEDING TO BE ENDOSCOPED WITHIN 24 HOURS OF ADMISSION - ARE WE MEETING BSG AND NICE GUIDELINES? THE EXPERIENCE OF A DISTRICT GENERAL HOSPITAL}

doi:10.1136/gutjnl-2013-304907.547

1J Barbour, ${ }^{1, *}$ S Smith, ${ }^{1} \mathrm{~K}$ St-Louis. 'Gateshead NHS Trust, Gateshead, UK

Introduction BSG and NICE guidelines recommend that all patients with a suspected GI bleed have an endoscopy within 24 hours of admission. The Queen Elizabeth Hospital (OEH), Gateshead (a District General Hospital serving a population of approximately 200,000) provides an urgent out of hours GI bleed service. Additionally there is an 8.30am slot in endoscopy from Monday to Friday allocated to patients who have symptoms of an upper GI bleed. We audited our compliance to the 24 hour guideline.

Methods The electronic reporting system 'Endosoft' was searched using the terms: Upper GI bleed; Malaena; or Haematemesis, for all referrals for gastroscopy between January $1^{\text {st }} 2011$ and December 31st 2011. The date, time of endoscopy, findings and interventions were noted. Time of admission, theatre use and In-patient and 4 week mortality were also noted.

Results 162 patients were admitted with acute GI bleeding over the 12 month period. Overall 126 (78\%) were endoscoped within 24 hours. 35 of the 36 patients endoscoped in theatre had their procedure within 24 hours of admission. 16 (44\%) of the 36 patients outside the 24 hour window were admitted on a Friday evening or Saturday. Of the 36 patients that suffered a delay to endoscopy: 5 had oesophageal varices; 1 a gastric varix; 6 peptic ulcer disease (4 high risk stigmata and 2 low risk); 2 Mallory Weiss tear, and 22 were normal. Of the 36 patients who went straight to theatre, 34 (94\%) had an endoscopic cause for bleeding identified. $20(56 \%)$ had variceal bleeds, 12 had peptic ulcer disease (10 high risk stigmata and 2 low risk). Overall in-patient mortality of those found to have an upper GI cause for bleeding was $16 \%$ (15 of 91 ) and 4 week mortality was 18\% (17 of 91). Five (29\%) of those that died were cirrhotic patients and $8(47 \%)$ had peptic ulcer disease with high risk stigmata. 3 of the 17 deaths occurred in the patients who were delayed prior to endoscopy, one of whom came in at a time when there was no allocated 8.30am slot the following morning. All 3 of the mortalities who waited over 24 hours for their procedure had endoscopic intervention. There was however no significant difference (chi-square test) between mortality of the delayed patients and those scoped within $24 \mathrm{hrs}$ (8\% compared to $11 \%$ ).

Conclusion During 2011 the $\mathrm{OEH}$ appropriately identified sick patients suitable for the out of hours GI bleed service, with $94 \%$ of theatre cases requiring endoscopic intervention. The NICE and BSG guidelines of endoscoping patients referred for a GI bleed within 24 hours of admission was met in $78 \%$ of cases. Allocated $8.30 \mathrm{am}$ endoscopy slots on Saturday and Sunday would improve these figures, but it is currently unclear if this would affect the patient outcome.

Disclosure of Interest None Declared.

\section{PTH-061 TO EVALUATE THE SAFETY AND OUTCOMES OF ESD AND HYBRID ESD FOR LARGE SESSILE COLORECTAL POLYPS INCLUDING PROCEDURAL COMPLICATIONS AND RECURRENCE RATE}

doi:10.1136/gutjnl-2013-304907.548

1.*S M Ali, 'N Radhakrishnan, 'R Hammonds, 'R George. 'Gastroenterology, Pennine Acute Hospitals NHS Trust, Manchester, UK

Introduction The purpose of ESD and Hybrid ESD (circumferential excision and snaring) is to obtain en bloc specimen. Margins are checked for residual tissue and APC applied if appropriate 
Methods Single endoscopist using ESD and Hybrid ESD (H-ESD) technique was retrospectively audited from April 2004 to August 2012. Service evaluation data of 38 patients with large sessile polyps who underwent ESD and Hybrid ESD was reviewed from a cohort of 224 colonoscopies referred for large polyp EMR. All procedures were intended as ESD. NICE recommendations for case selection were followed in $92 \%$ cases. Due to challenges in submucosal dissection of the large lesions, piecemeal resection was done after circumferential cutting. First follow-up endoscopy was performed at 3-6 months and the second at 12-14 months.

Results Mean age was 70 with 16 males and 22 females. Mean size of polyp in ESD group was $26 \mathrm{~mm}$. Range $15-50 \mathrm{~mm}$. Mean size in the H-ESD group was $49 \mathrm{~mm}$. Range $20-100 \mathrm{~mm}$. Complete resections were achieved in 17 (44\%) out of 38 cases. Due to piecemeal resection pathologists were not able to confirm adequacy of excision in 12 cases. In 9 cases resection was reported incomplete on index procedure. ESD performed in 13 (34\%) cases. Complete resection achieved in 6 . Out of 7 incomplete resections in the ESD group, 3 were reported by pathologists as lesion extending to the lateral margin hence incomplete excision. Histology did not comment on margin clearance in 3 ESD. 1 ESD was a sub mucosal lipoma on histology. This was an unintentional ESD for lipoma. Histology: ESD group: TVA with LGD 7, TVA with HGD 5. H-ESD was performed in $25(65.7 \%)$ cases. Complete resection achieved in 11 cases, incomplete resection in 7 and lateral margin clearance not confirmed in $7 \mathrm{H}$-ESD cases due to piecemeal resection. Histology: H-ESD group: TVA with LGD 16, TVA with HGD 6 and adenocarcinoma in 2 cases-one's lateral and deep margins were clear and the other was incomplete and referred to MDT In $4 \mathrm{ESD}$ and $7 \mathrm{H}-\mathrm{ESD}$ cases there was minor bleeding controlled endoscopically at the time. 1 delayed post- H-ESD bleeding required 11 days of hospital stay and 2 units of blood transfusion. 1 retroperitoneal perforation and 1 case of serosal cut managed conservatively with clips and antibiotics. APC performed in 16 $(42 \%)$ out of 38 . Recurrence was identified in $6 \mathrm{H}$-ESD cases (15.7\%). 5 local recurrences detected at 3 months and 1 local recurrence detected at 24 months. In 13 ESD cases no perforation or recurrence upto 14 months was noted despite 5 histologically incomplete dissections.

Conclusion ESD in bowel is challenging and has a long learning curve. These procedures should be performed by trained endoscopists in accredited units and a national registry should be maintained.

Disclosure of Interest None Declared.

\section{PTH-062 ENDOSCOPIC MUCOSAL RESECTION OF LARGE COLORECTAL POLYPS IN NON SCREENING COLONOSCOPIES HAS A HIGH RECURRENCE RATE:}

doi:10.1136/gutjnl-2013-304907.549

1,"S M Ali, 'R Kalla, 'M Hendrickse. 'Gastroenterology, Blackpool Victoria Hospital, Blackpool, UK

Introduction EMR of large colorectal polyps has been reported to be a safe and effective technique in UK. Although the success of large polyp EMR has been reported within regional bowel cancer screening centres, there is a paucity of data comparing outcomes between screening and non-screening centres.

Methods A total of 61 screening and 60 non-screening EMR of polyps $2 \mathrm{~cm}$ or greater were performed from January 2008 to December 2011. Data was collected from 3 hospitals for screening EMR and 1 district hospital for non-screening EMR.

Results The table below summarises the findings at initial EMR for both groups.

\section{Abstract PTH-062 Table 1}

\begin{tabular}{lcc}
\hline Characteristics & $\begin{array}{c}\text { Results for Screening } \\
\text { group n (\%) }\end{array}$ & $\begin{array}{c}\text { Results for non-screening } \\
\text { group } \mathbf{n}(\%)\end{array}$ \\
\hline Number of patients & 61 & 60 \\
Mean size (in mm) & 35 & 43 \\
Location (Left colon) & $43(70)$ & $36(60)$ \\
Histology (Low Grade Dysplasia) & $47(83)$ & $36(67)$ \\
Histology (High Grade Dysplasia) & $7(12)$ & $11(20)$ \\
Histology (Adenocarcinoma) & $3(5)$ & $7(13)$ \\
Bleeding Complication & $2(3)$ & $3(5)$ \\
Perforation & $0(0)$ & $1(2)$ \\
\hline
\end{tabular}

Surveillance data was available for 37 patients in the non-screening group compared to all 61 patients in the screening group up-to 3 months post EMR. Number of recurrences were $6(10 \%)$ with mean polyp size of $25 \mathrm{~mm}$ in the screening group compared to $10(27 \%)$ in the non screening group with mean polyp size of $50 \mathrm{~mm}$.

All recurrences in both groups were resected and complete. Histology confirmed low grade dysplasia (LGD) in $80 \%$, while 2 patients had evidence of high grade dysplasia (HGD) in the non-screening group. All showed LGD in the screening group. At 12 months surveillance post EMR, 43 screening patients had follow-up data with 8 recurrences detected compared to 14 in the non screening group with 4 recurrences.

Conclusion Endoscopic mucosal resection of polyps in screening group appears to be safer with low complication rates and low recurrence rate up to 12 months post EMR compared to the nonscreening groups. This may be due to a multitude of factors such as patient age, co-morbidites and the characteristics of polyps. Clinical features of patients undergoing EMR were similar in both groups. There was a trend to higher recurrence and complication rate in the non screening group. The follow up post EMR was less consistent in the non screening group possibly due to less stringent protocols for follow up. This may have affected the surveillance and recurrence rates.

Disclosure of Interest None Declared.

\section{REFERENCES}

Lim TR, Mahesh V, Singh S, et al. Endoscopic mucosal resection of colorectal polyps in typical UK hospitals. World J Gastroenterol.2010; 16(42): 5324-8.

Boix J, Lorenzo-Zúñiga V, Moreno de Vega V, et al. Endoscopic removal of large sessile colorectal adenomas: is it safe and effective? Dig Dis Sci 2007; 52 840-844

Salama M, Ormonde D, Quach T, et al. Outcomes of endoscopic resection of large colorectal neoplasms: an Australian experience. J Gastroenterol Hepatol 2010; 25 $84-89$

\section{PTH-063 ESTABLISHING AUDIT STANDARDS FOR COLONIC STENT INSERTION WILL FACILITATE SERVICE EVALUATION AND PLANNING FOR A RAPIDLY GROWING SERVICE}

doi:10.1136/gutjnl-2013-304907.550

1,*T M Cacciottolo, ' $\mathrm{D}$ Menzies, 'A Shenoy, 'D 0'Riordan, 'I Gooding. 'Gastroenterology; ${ }^{2}$ Surgery, Colchester General Hospital, Colchester, UK

Introduction Self-expandable metal stent (SEM) placement is the recommended treatment option by the National Institute for Clinical Excellence (NICE) for acute colorectal obstruction in the palliative management of inoperable colorectal cancer (CRC) as well as a bridge to planned single stage surgical intervention[1]. NICE guidance recommends that centres offering this treatment modality should have teams with expertise and capacity to stent 15 people per million population per annum[2]. It does not however provide any suitable standard for audit. 